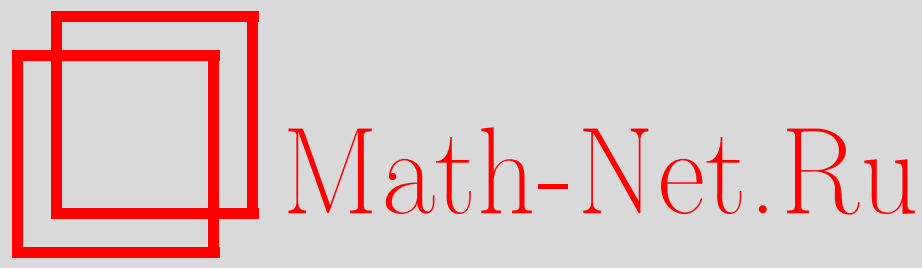

В. В. Вальков, Д. М. Дзебисашвили, Эффективные взаимодействия в периодической модели Андерсона в режиме смешанной валентности при сильных корреляциях, TMФ, 2008, том 157, номер 2, 235-249

DOI: https://doi.org/10.4213/tmf6277

Использование Общероссийского математического портала Math-Net.Ru подразумевает, что вы прочитали и согласны с пользовательским соглашением http://www . mathnet.ru/rus/agreement

Параметры загрузки:

IP: 54.198 .55 .26

26 апреля 2023 г., 12:58:18

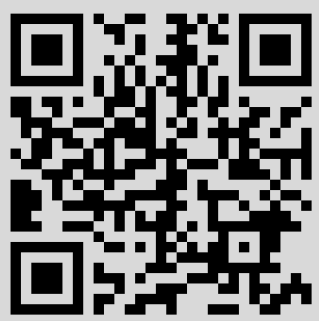




\title{
ЭФФЕКТИВНЫЕ ВЗАИМОДЕЙСТВИЯ В ПЕРИОДИЧЕСКОЙ МОДЕЛИ АНДЕРСОНА В РЕЖИМЕ СМЕШАННОЙ ВАЛЕНТНОСТИ ПРИ СИЛЬНЫХ КОРРЕЛЯЦИЯХ
}

\begin{abstract}
Для периодической модели Андерсона в режиме сильных корреляций с точностью до членов четвертого порядка по параметру $V / U(V-$ интенсивность гибридизационного взаимодействия, $U$ - величина внутриатомного кулоновского отталкивания) построен эффективный гамильтониан $H_{\text {eff }}$, содержащий взаимодействия, индуцирующие как магнитное упорядочение, так и куперовскую неустойчивость в условиях смешанной валентности редкоземельных ионов. На основе численных расчетов получены данные о зависимости параметров эффективных взаимодействий от расстояния между узлами кристаллической решетки. Показано, что реализация обменных связей соответствует сильно фрустрированной системе локализованных спиновых моментов и способствует подавлению антиферромагнитного параметра порядка с возможным переходом в состояние квантовой спиновой жидкости. Существенно, что среди слагаемых $H_{\text {eff }}$, индуцирующих переход в сверхпроводящую фазу, содержатся слагаемые, приводящие к $d$-типу симметрии сверхпроводящего параметра порядка, который реализуется во многих тяжелофермионных соединениях.
\end{abstract}

Ключевые слова: периодическая модель Андерсона, смешанная валентность, тяжелые фермионы, эффективный гамильтониан, сверхпроводимость.

\section{1. ВВЕДЕНИЕ}

Периодическая модель Андерсона (ПАМ) активно используется на протяжении нескольких десятков лет для теоретического исследования необычных свойств интерметаллидов, содержащих ионы редкоземельных и актинидных элементов (см., например, сборники [1]). В зависимости от соотношения между параметрами модели (положения локализованного уровня $E_{f}$, ширины зоны коллективизированных электронов $W$, интенсивности гибридизационного взаимодействия $V$ и величины внутриатомного кулоновского отталкивания $U$ ) реализуется качественно различный тип основного состояния системы [2]. Если ввести безразмерную обменную

${ }^{*}$ Институт физики им. Л. В. Киренского СО РАН, Сибирский федеральный университет, Красноярск, Россия. E-mail: vvv@iph.krasn.ru; ddm@iph.krasn.ru 
константу связи между спиновыми моментами локализованных и коллективизированных электронов $g=2 V^{2} \rho_{\mathrm{c}}(\mu)\left|E_{f}-\mu\right|^{-1}$ (здесь $\mu$ - химический потенциал, $\rho_{\mathrm{c}}(\mu)-$ плотность состояний электронов проводимости на уровне Ферми) [3], то при $g>1$ реализуется состояние промежуточной валентности, а при $g<1$ система находится либо в тяжелофермионном режиме Кондо, при котором в области низких температур значения электронной теплоемкости и магнитной восприимчивости аномально велики, либо в магнитной фазе, в которой РККИ-взаимодействие подавляет кондовские флуктуации.

При исследовании низкоэнергетической части спектра элементарных возбуждений ПАМ часто используется переход к эффективному гамильтониану $H_{\text {eff }}[4]-[6]$. Целью такого перехода является получение эффективных взаимодействий, ответственных за формирование того или иного типа основного состояния и спектра элементарных возбуждений. Например, для изучения условий возникновения дальнего магнитного порядка и магнонных ветвей спектра актуальными являются обменное и $s-f$-обменное взаимодействия. Как известно, эти взаимодействия можно получить в эффективном гамильтониане ПАМ во втором и четвертом порядках по параметру

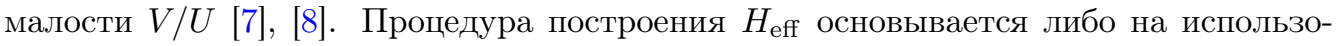
вании метода унитарных преобразований [7]-[12] (при унитарных преобразованиях энергетический спектр гамильтониана не меняется), либо на применении операторной формы теории возмущений [13], [14]. Структура получаемого эффективного гамильтониана зависит также от представления операторов, т.е. от того, используются фермиевские операторы вторичного квантования или операторы Хаббарда, удовлетворяющие более сложным правилам коммутации (или антикоммутации).

В работе [10] при выводе низкоэнергетического гамильтониана ПАМ рассматривалось следующее соотношение между параметрами модели: $W \ll V \ll U$. Для получения $H_{\mathrm{eff}}$, во-первых, применялось унитарное преобразование, диагонализующее одноионную часть ПАМ, записанную в представлении операторов Хаббарда, во-вторых, результат преобразования проектировался на низкоэнергетическое подпространство состояний, отделенное от возбужденных на величину порядка $U$, в-третьих, применялось обратное унитарное преобразование. Полученный таким образом эффективный гамильтониан позволил авторам исследовать вопрос о существовании связанных парных состояний в ПАМ. В работе [9] построен гамильтониан до четвертого порядка по $V / U$ путем последовательного применения двух унитарных преобразований, исключающих гибридизационные переходы в первом и третьем порядках. Главный момент подхода данной работы заключался в том, что гамильтониан ПАМ сначала проектировался на нижнюю хаббардовскую подзону (т.е. на гильбертово подпространство локализованных состояний, не содержащее два электрона), и лишь затем унитарными преобразованиями исключались гибридизационные процессы, идущие в нижней хаббардовской подзоне.

Унитарные преобразования $H \longrightarrow \widetilde{H}=e^{S} H e^{-S}$ в большинстве случаев проводятся при условии, что антиэрмитовый оператор $S$ записывается через комбинацию фермиевских операторов. Это позволяет в режиме Кондо легко устранить в первом порядке гибридизационные процессы между двумя подгруппами электронов и получить эффективный гамильтониан, описывающий, в частности, обменную связь 
между спиновыми моментами отмеченных групп электронов. Однако, как известно, такой подход не пригоден, если локализованный уровень находится в континууме коллективизированных состояний и лежит в непосредственной близости от уровня Ферми. Как показано в работе [12], в этом случае значения обменных интегралов расходятся. Между тем именно этот режим является особенно интересным как с точки зрения объяснения магнитных свойств части редкоземельных и актинидных интерметаллидов, так и с точки зрения получения явного вида эффективных взаимодействий, ответственных за формирование куперовской неустойчивости в системе тяжелых фермионов. Кроме того, также представляется актуальной задача построения в режиме промежуточной валентности эффективного гамильтониана, описывающего антиферромагнитные взаимодействия, существенно влияющие на формирование сверхпроводящей фазы с $d$-типом симметрии параметра порядка. Рассмотрению этих вопросов посвящена настоящая работа.

Ключевой момент предлагаемого метода заключается в разбиении оператора гибридизационного взаимодействия в атомном представлении на два слагаемых. Первое слагаемое отражает процессы, приводящие к смешиванию коллективизированных и локализованных электронов без изменения числа состояний, называемых двойками, в локализованной подсистеме (низкоэнергетический сектор гильбертова пространства). Второе слагаемое описывает гибридизационные процессы с одновременным рождением (либо уничтожением) двоек и явно учитывает процессы, индуцирующие переходы в высокоэнергетический сектор гильбертова пространства. Вследствие того, что энергетический параметр $U$ велик, эти процессы удается учесть по теории возмущений, получив аналитическое разложение по степеням малого параметра (конкретные условия применимости разложения см. ниже). В результате возникает эффективный гамильтониан, описывающий не только обменные взаимодействия, но и взаимодействия, явно указывающие на возможность куперовской неустойчивости и образование сверхпроводящей фазы с развитыми антиферромагнитными флуктуациями.

\section{2. УНИТАРНЫЕ ПРЕОБРАЗОВАНИЯ ГАМИЛЬТОНИАНА}

Воспользовавшись атомным представлением и разбив оператор гибридизационного взаимодействия на два слагаемых, запишем гамильтониан ПАМ в виде

$$
H=H_{0}+V_{01}+V_{12},
$$

где

$$
\begin{aligned}
H_{0} & =\sum_{k \sigma}\left(\varepsilon_{k}-\mu\right) c_{k \sigma}^{+} c_{k \sigma}+\sum_{f \sigma}\left(E_{0}-\mu\right) X_{f}^{\sigma \sigma}+\sum_{f}\left(2 E_{0}-2 \mu+U\right) X_{f}^{22}, \\
V_{01} & =\frac{1}{\sqrt{N}} \sum_{k f \sigma}\left(V_{k} e^{-i k f} c_{k \sigma}^{+} X_{f}^{0 \sigma}+V_{k}^{*} e^{i k f} X_{f}^{\sigma 0} c_{k \sigma}\right), \\
V_{12} & =\frac{1}{\sqrt{N}} \sum_{k f \sigma} 2 \sigma\left(V_{k} e^{-i k f} c_{k \sigma}^{+} X_{f}^{\bar{\sigma} 2}+V_{k}^{*} e^{i k f} X_{f}^{2 \bar{\sigma}} c_{k \sigma}\right) .
\end{aligned}
$$


Здесь $c_{k \sigma}^{+}\left(c_{k \sigma}\right)$ - оператор рождения (уничтожения) коллективизированного электрона с импульсом $k$, проекцией спинового момента $\sigma= \pm 1 / 2$ и энергией $\varepsilon_{k}$. Операторы Хаббарда действуют в подпространстве локализованных состояний и определяются обычным образом: $X_{f}^{n, m}=|f, n\rangle\langle m, f|$. Каждый из индексов одноузельного локализованного состояния может принимать одно из четырех значений: $m, n \in$ $\{0, \uparrow, \downarrow, 2\}$. Индекс ноль используется для описания одноузельного состояния без электронов. Векторам $|f, \uparrow\rangle$ и $|f, \downarrow\rangle$ отвечают состояния с одним электроном на узле $f$ и проекцией спинового момента, направленной вдоль и против оси квантования соответственно. Эти состояния обладают затравочной энергией $E_{0}$. Вектор $|f, 2\rangle$ описывает состояния с двумя электронами на узле $f$ (так называемые двойки). При учете кулоновского взаимодействия $U$ затравочная энергия такого состояния определяется выражением $2 E_{0}+U$. Диагональный оператор Хаббарда $X_{f}^{n n}$ является проекционным оператором, а недиагональный $X_{f}^{n m}, n \neq m$, описывает переход из одноузельного состояния $|f, m\rangle$ в состояние $|f, n\rangle$. Через $V_{k}$ обозначен фурье-образ гибридизационного взаимодействия. Для дальнейшего изложения существенно, что оператор $\widehat{V}_{12}$, в отличие от оператора $\widehat{V}_{01}$, описывает процессы гибридизационного смешивания с одновременным индуцированием (аннигилированием) двоечных состояний из высокоэнергетического сектора гильбертова пространства.

Если параметры модели такие, как показано на рис. 1 , причем $\left|V_{k}\right| \ll E_{0}+U-\varepsilon_{\max }$, $\left(\varepsilon_{\max }\right.$ - максимальное значение энергии электрона проводимости), то вклады от оператора $\widehat{V}_{12}$ можно учесть по теории возмущений. С этой целью, используя указанное неравенство, построим эффективный гамильтониан, применяя ряд последовательных унитарных преобразований.

На первом этапе выполним унитарное преобразование $H \longrightarrow \widetilde{H}=e^{S} H e^{-S}$ с таким $S$, чтобы в разложении $\widetilde{H}$ в первом порядке не содержался оператор $V_{12}$. Для этого потребуем выполнения операторного равенства

$$
\left[S, H_{0}\right]=-V_{12}
$$

определяющего $S$. После простых вычислений находим

$$
S=-\frac{1}{\sqrt{N}} \sum_{k f \sigma} 2 \sigma\left(\eta_{k} e^{-i k f} c_{k \sigma}^{+} X_{f}^{\bar{\sigma} 2}-\eta_{k}^{*} e^{i k f} X_{f}^{2 \bar{\sigma}} c_{k \sigma}\right)
$$

где безразмерный параметр

$$
\eta_{k}=\frac{V_{k}}{U+E_{0}-\varepsilon_{k}} \ll 1
$$

Разложив $\widetilde{H}=e^{S} H e^{-S}$ в ряд по параметру $\eta_{k}$ и ограничиваясь слагаемыми до четвертого порядка малости включительно, получим, что $\widetilde{H}$ определяется выражением

$$
\widetilde{H}=H_{0}+V_{01}+\left[S,\left(V_{01}+\frac{V_{12}}{2}\right)\right]+\left[S,\left[S,\left(\frac{V_{01}}{2}+\frac{V_{12}}{3}\right)\right]\right]+\left[S,\left[S,\left[S, \frac{V_{01}}{6}+\frac{V_{12}}{8}\right]\right]\right] .
$$

Заметим, что в полученном выражении для $\widetilde{H}$, начиная с членов второго порядка по параметру $\eta_{k}$, вновь содержатся операторы, отражающие гибридизационные 


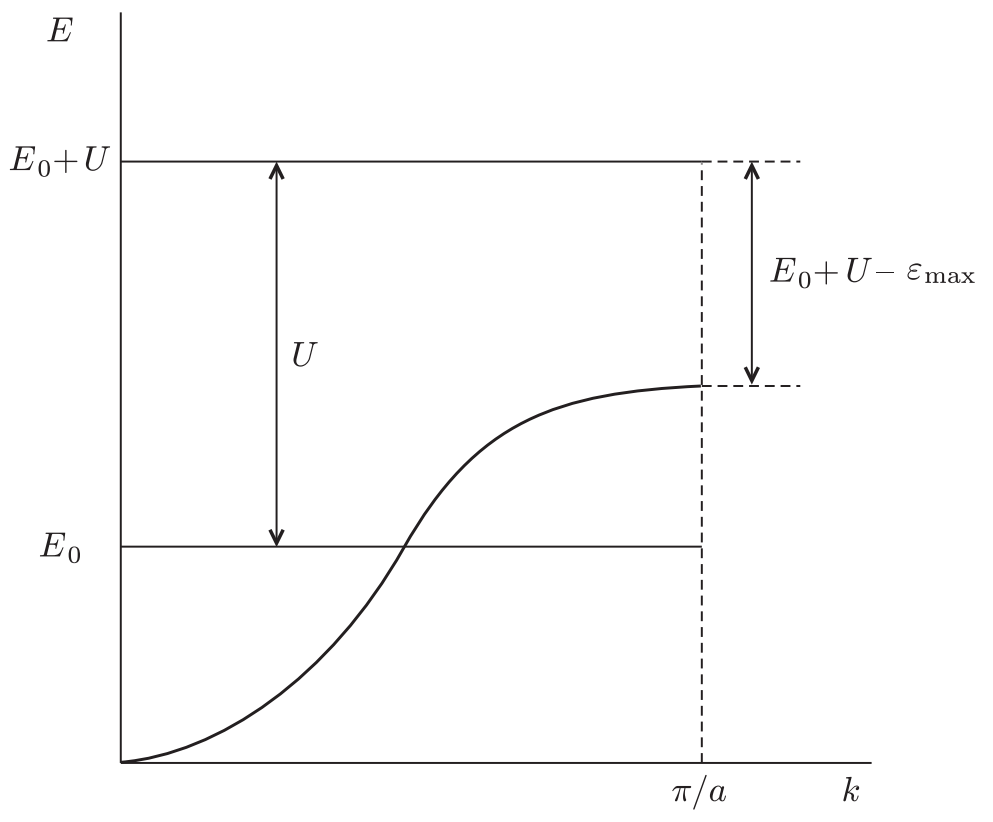

Рис. 1. Затравочная энергетическая структура периодической модели Андерсона.

процессы с участием высокоэнергетического сектора гильбертова пространства. Это означает, что такие операторы будут приводить к дополнительным вкладам четвертого порядка в эффективный гамильтониан. Для расчета соответствующих операторных выражений приходится использовать многоэтапную процедуру проведения унитарных преобразований (см. ниже).

Вычисляя первые коммутаторы, получаем, что вклад второго порядка в $\widetilde{H}$ определяется выражением

$$
\begin{array}{r}
\widetilde{H}_{(2)}=\sum_{f m \sigma} 2 \sigma\left(t_{f m} X_{f}^{\sigma 0} X_{m}^{\bar{\sigma} 2}+\text { э.c. }\right)+\frac{1}{N} \sum_{k p f}\left[\Delta_{f}(k, p)\left(c_{k \uparrow}^{+} c_{p \downarrow}^{+}-c_{k \downarrow}^{+} c_{p \uparrow}^{+}\right) X_{f}^{02}+\text { э.c. }\right]+ \\
+\sum_{f m \sigma} t_{f m} X_{f}^{2 \sigma} X_{m}^{\sigma 2}+\frac{1}{N} \sum_{q p f \sigma} A_{f}(q, p)\left(X_{f}^{\bar{\sigma} \sigma} c_{q \sigma}^{+} c_{p \bar{\sigma}}-\left(X_{f}^{\bar{\sigma} \bar{\sigma}}+X_{f}^{22}\right) c_{q \sigma}^{+} c_{p \sigma}\right) .
\end{array}
$$

Возникающие в этом соотношении операторные слагаемые описывают четыре типа взаимодействий. Первый тип взаимодействия в атомном представлении проявляется как совокупность квантовых переходов между нижней и верхней хаббардовскими подзонами. Интенсивность этих переходов определяется матричным элементом

$$
t_{f m}=\frac{1}{N} \sum_{q} \eta_{q} V_{q}^{*} e^{i q(f-m)} .
$$

Второй тип взаимодействия связан с процессами рождения (уничтожения) двоек в локализованной подсистеме и одновременным уничтожением (рождением) синглет- 
ной пары коллективизированных электронов с не обязательно равным нулю импульсом центра масс. Интенсивность таких процессов задается величиной

$$
\Delta_{f}(k, p)=\eta_{k} V_{p} e^{-i f(k+p)} .
$$

Третий тип взаимодействия проявляется посредством перескоков $f$-электронов в верхней хаббардовской подзоне с матричным элементом $t_{f m}$. Наконец, последний тип взаимодействия описывает обменную связь и кулоновские корреляции между двумя подсистемами электронов. Интенсивность таких процессов пропорциональна

$$
A_{f}(q, p)=\frac{1}{2} e^{-i f(q-p)}\left[\eta_{q} V_{p}^{*}+\eta_{p}^{*} V_{q}\right] .
$$

При записи вкладов от коммутаторов более высокого порядка учтем, что конечная цель вычислений заключается в построении эффективного гамильтониана $H_{\text {eff }}$ с точностью до членов четвертого порядка малости включительно. Существенно, что сектор гильбертова пространства без двоек должен быть инвариантным относительно действия в нем $H_{\text {eff }}$. Если ввести оператор проецирования на этот сектор

$$
P=\prod_{f}\left(X_{f}^{00}+X_{f}^{\uparrow \uparrow}+X_{f}^{\downarrow \downarrow}\right),
$$

то указанное требование математически записывается как

$$
P H_{\mathrm{eff}} P=P H_{\mathrm{eff}}=H_{\mathrm{eff}} P=H_{\mathrm{eff}} .
$$

Следовательно, окончательный вклад от коммутаторов третьего и четвертого порядков определяется только спроецированными на не содержащий двоек сектор слагаемыми. Как нетрудно убедиться, $P\left[S,\left[S, V_{12}\right]\right] P=0$ и $P\left[S,\left[S,\left[S, V_{01}\right]\right]\right] P=0$, поэтому выражения для $\widetilde{H}_{(3)}$ и $\widetilde{H}_{(4)}$, определяемые вкладами от оставшихся коммутаторов, имеют вид

$$
\begin{aligned}
& \widetilde{H}_{(3)}=\frac{1}{2} P\left[S,\left[S, V_{01}\right]\right] P=P \frac{1}{2 \sqrt{N}} \sum_{f m k \sigma}\left[\eta_{k} t_{f m} e^{-i k f}\left(c_{k \bar{\sigma}}^{+} X_{f}^{\sigma \bar{\sigma}}-c_{k \sigma}^{+} X_{f}^{\bar{\sigma} \bar{\sigma}}\right) X_{m}^{0 \sigma}+\text { э.c. }\right]+ \\
& +P \frac{1}{2 N^{3 / 2}} \sum_{k q p f \sigma} 2 \sigma\left[\eta_{q} e^{-i q f} \Delta_{f}^{*}(k, p) X_{f}^{\bar{\sigma} 0} c_{q \sigma}^{+}\left(c_{k \downarrow} c_{p \uparrow}-c_{k \uparrow} c_{p \downarrow}\right)+\text { э.c. }\right], \\
& \widetilde{H}_{(4)}=\frac{1}{8} P\left[S,\left[S,\left[S, V_{12}\right]\right]\right] P= \\
& =P \frac{1}{8 N} \sum_{k q f m \sigma}\left[\eta_{q} B_{k}^{*}(f, m) e^{-i q m}\left(c_{q \sigma}^{+} X_{m}^{\bar{\sigma} \bar{\sigma}}-c_{q \bar{\sigma}}^{+} X_{m}^{\sigma \bar{\sigma}}\right)\left(c_{k \sigma} X_{f}^{\bar{\sigma} \bar{\sigma}}-c_{k \bar{\sigma}} X_{f}^{\bar{\sigma} \sigma}\right)+\text { э.c. }\right]+ \\
& +P \frac{1}{4 N^{2}} \sum_{p k q k_{1} f \sigma}\left[\eta_{k_{1}} C_{f}^{*}(k, q, p) e^{-i k_{1} f} c_{p \bar{\sigma}}^{+}\left(c_{k_{1} \sigma}^{+} X_{f}^{\bar{\sigma} \bar{\sigma}}-c_{k_{1} \bar{\sigma}}^{+} X_{f}^{\sigma \bar{\sigma}}\right) c_{k \bar{\sigma}} c_{q \sigma}+\text { э.c. }\right] .
\end{aligned}
$$

Входящие в последние равенства функции определены следующим образом:

$$
\begin{aligned}
B_{k}(f, m) & =2 \eta_{k} t_{f m} e^{-i k f}+\frac{2}{N} \sum_{p} \eta_{p} A_{f}(k, p) e^{-i p m}, \\
C_{f}(k, q, p) & =\eta_{k} A_{f}(q, p) e^{-i k f}+\eta_{q} A_{f}(k, p) e^{-i q f} .
\end{aligned}
$$


Собирая вместе полученные выражения, находим $\widetilde{H}$ :

$$
\widetilde{H}=H_{0}+V_{01}+\widetilde{H}_{(2)}+\widetilde{H}_{(3)}+\widetilde{H}_{(4)}
$$

Из выражения (7) видно, что две первые суммы в правой части этого равенства описывают процессы, приводящие к изменению числа двоек в системе. Поскольку эти операторы имеют второй порядок малости, процессы генерации двоек с последующей их аннигиляцией приведут к дополнительному взаимодействию четвертого порядка. Для нахождения вкладов отмеченных процессов сделаем второе унитарное преобразование, которое с рассматриваемой точностью не изменяет операторов $\widetilde{H}_{(3)}$ и $\widetilde{H}_{(4)}$. Тогда искомый эффективный гамильтониан $H_{\text {eff }}$ определяется следующим выражением:

$$
H_{\mathrm{eff}}=P e^{S_{1}} \widetilde{H} e^{S_{1}} P
$$

Оператор $S_{1}$, задающий унитарное преобразование, выбирается так, чтобы в выражении $e^{S_{1}} \widetilde{H} e^{S_{1}}$ были исключены процессы второго порядка, генерирующие двойки. Этого можно достичь, если $S_{1}$ записать в виде

$$
\begin{aligned}
S_{1}=\{ & \frac{1}{N} \sum_{k p f}\left[\frac{\Delta_{f}(k, p)}{\varepsilon_{k}+\varepsilon_{p}-U-2 E_{0}}\right]\left(c_{k \uparrow}^{+} c_{p \downarrow}^{+}-c_{k \downarrow}^{+} c_{p \uparrow}^{+}\right) X_{f}^{02}- \\
& \left.-\sum_{g m \sigma} 2 \sigma\left(\frac{t_{g m}}{U}\right) X_{g}^{\sigma 0} X_{m}^{\bar{\sigma} 2}\right\}-\{\text { э.c. }\} .
\end{aligned}
$$

После вычисления коммутаторов и проецирования на сектор гильбертова пространства, не содержащий двоек, получаем окончательный вид эффективного гамильтониана:

$$
\begin{aligned}
& H_{\mathrm{eff}}=\sum_{k \sigma}\left(\varepsilon_{k}-\mu\right) c_{k \sigma}^{+} c_{k \sigma}+\sum_{f \sigma}\left(E_{0}-\mu\right) X_{f}^{\sigma \sigma}+\frac{1}{\sqrt{N}} \sum_{k f \sigma}\left[V_{k} e^{-i k f} c_{k \sigma}^{+} X_{f}^{0 \sigma}+\text { э.c. }\right]+ \\
& +\frac{1}{N} \sum_{q p f \sigma} A_{f}(q, p)\left[c_{q \bar{\sigma}}^{+} X_{f}^{\sigma \bar{\sigma}}-c_{q \sigma}^{+} X_{f}^{\bar{\sigma} \bar{\sigma}}\right] c_{p \sigma}+ \\
& +\frac{1}{2 \sqrt{N}} \sum_{f m k \sigma}\left[\eta_{k} t_{f m} e^{-i k f}\left(c_{k \bar{\sigma}}^{+} X_{f}^{\sigma \bar{\sigma}}-c_{k \sigma}^{+} X_{f}^{\bar{\sigma} \bar{\sigma}}\right) X_{m}^{0 \sigma}+\text { э.c. }\right]+ \\
& +\frac{1}{2 N^{3 / 2}} \sum_{k q p f}\left[\eta_{q} \Delta_{f}^{*}(k, p) e^{-i q f}\left(X_{f}^{\downarrow 0} c_{q \uparrow}^{+}-X_{f}^{\uparrow 0} c_{q \downarrow}^{+}\right)\left(c_{k \downarrow} c_{p \uparrow}-c_{k \uparrow} c_{p \downarrow}\right)+\text { э.c. }\right]+ \\
& +\frac{1}{N} \sum_{k q f m \sigma}\left[\frac{1}{8} \eta_{q} B_{k}^{*}(f, m) e^{-i q m}\left(c_{q \bar{\sigma}}^{+} X_{m}^{\sigma \bar{\sigma}}-c_{q \sigma}^{+} X_{m}^{\bar{\sigma} \bar{\sigma}}\right)\left(c_{k \bar{\sigma}} X_{f}^{\bar{\sigma} \sigma}-c_{k \sigma} X_{f}^{\bar{\sigma} \bar{\sigma}}\right)+\text { э.c. }\right]- \\
& -\frac{1}{N^{2}} \sum_{p k q k_{1} f \sigma}\left[\frac{1}{4} \eta_{k_{1}} C_{f}^{*}(k, q, p) e^{-i k_{1} f} c_{p \bar{\sigma}}^{+}\left(c_{k_{1} \bar{\sigma}}^{+} X_{f}^{\sigma \bar{\sigma}}-c_{k_{1} \sigma}^{+} X_{f}^{\bar{\sigma} \bar{\sigma}}\right) c_{k \bar{\sigma}} c_{q \sigma}+\text { э.c. }\right]+ \\
& +\frac{1}{N} \sum_{k p f m}\left\{\left[\frac{\Delta_{f}(k, p)}{\varepsilon_{k}+\varepsilon_{p}-U-2 E_{0}}\right] t_{f m}\left(c_{k \uparrow}^{+} c_{p \downarrow}^{+}-c_{k \downarrow}^{+} c_{p \uparrow}^{+}\right)\left(X_{f}^{0 \downarrow} X_{m}^{0 \uparrow}-X_{f}^{0 \uparrow} X_{m}^{0 \downarrow}\right)+\text { э.c. }\right\}+
\end{aligned}
$$




$$
\begin{aligned}
& +\frac{1}{N^{2}} \sum_{k p f k_{1} p_{1}}\left[\frac{\Delta_{f}(k, p) \Delta_{f}^{*}\left(k_{1}, p_{1}\right)}{\varepsilon_{k}+\varepsilon_{p}-U-2 E_{0}}\right] X_{f}^{00}\left(c_{k \uparrow}^{+} c_{p \downarrow}^{+}-c_{k \downarrow}^{+} c_{p \uparrow}^{+}\right)\left(c_{p_{1} \downarrow} c_{k_{1} \uparrow}-c_{p_{1} \uparrow} c_{k_{1} \downarrow}\right)+ \\
& +\sum_{f m} J_{f m}\left(\vec{S}_{f} \vec{S}_{m}-\frac{1}{4} \widehat{N}_{f} \widehat{N}_{m}\right)+\sum_{\substack{f m g \sigma \\
f \neq g)}}\left(\frac{t_{f m} t_{m g}}{U}\right)\left(X_{f}^{\sigma 0} X_{m}^{\bar{\sigma} \sigma} X_{g}^{0 \bar{\sigma}}-X_{f}^{\sigma 0} X_{m}^{\bar{\sigma} \bar{\sigma}} X_{g}^{0 \sigma}\right),
\end{aligned}
$$

где $\vec{S}_{f}$ является квазиспиновым векторным оператором с компонентами

$$
\begin{gathered}
S_{f}^{x}=\frac{S_{f}^{+}+S_{f}^{-}}{2}, \quad S_{f}^{y}=\frac{S_{f}^{+}-S_{f}^{-}}{2 i}, \quad S_{f}^{z}=\frac{X_{f}^{\uparrow \uparrow}-X_{f}^{\downarrow \downarrow}}{2}, \\
S_{f}^{+}=X_{f}^{\uparrow \downarrow}, \quad S_{f}^{-}=X_{f}^{\downarrow \uparrow} .
\end{gathered}
$$

Квазиспиновый характер операторов $S_{f}^{x}, S_{f}^{y}, S_{f}^{z}$ связан с тем, что среди допустимых состояний на узле $f$ могут быть состояния без электрона. Поэтому несмотря на строгую выполнимость коммутационных соотношений, соответствующих спиновым операторам, операторы $S_{f}^{x}, S_{f}^{y}, S_{f}^{z}$ не могут быть отождествлены со спиновыми операторами. Это следует, например, из того, что $\left(\vec{S}_{f}\right)^{2}=(3 / 4) \widehat{N_{f}}$, и только в предельном случае, когда на каждом узле $f$ находится точно по одному электрону, квазиспиновые операторы становятся строго спиновыми. Оператор $\widehat{N}_{f}=\sum_{\sigma} X_{f}^{\sigma \sigma}$ является оператором числа локализованных электронов на узле $f$. Интеграл обменного взаимодействия $J_{f m}=2\left(t_{f m} t_{m f}\right) / U$ имеет происхождение, аналогичное происхождению обменной связи в $t-J$-модели [15]. В окончательном выражении для эффективного гамильтониана отсутствует оператор проецирования, поскольку интересующий нас низкоэнергетический сектор гильбертова пространства инвариантен относительно действия $H_{\text {eff }}$.

\section{3. ИЕРАРХИЯ ЭФФЕКТИВНЫХ ВЗАИМОДЕЙСТВИЙ}

Остановимся на физическом содержании слагаемых эффективного гамильтониана (19) ПАМ. Первые два члена гамильтониана описывают невзаимодействующие подсистемы коллективизированных и локализованных электронов. Для локализованных электронов используется атомное представление с гильбертовым пространством, не содержащим двоек, поэтому в дальнейшем эти электроны мы будем называть фермионами Хаббарда. Третье слагаемое описывает гибридизацию между двумя коллективизированными электронами и фермионами Хаббарда.

Во втором порядке по $V_{k}$ имеется лишь одно слагаемое, интенсивность которого определяется матричным элементом $A_{f}(q, p)$. Оно описывает рассеяние электронов проводимости на локализованных $f$-состояниях. Часть таких взаимодействий сводится к обменному $s-f$-взаимодействию, способному, как известно, индуцировать синглетное состояние $f$ - и $c$-электронов и экранировать локализованные магнитные моменты (эффект Кондо).

Процессы взаимодействия, имеющие третий порядок по $V_{k}$, описываются двумя слагаемыми. Первое из них, пропорциональное $\eta_{k} t_{f m}$, описывает коррелированные с локализованными состояниями гибридизационные переходы между двумя группами фермионов. Второе слагаемое описывает процессы уничтожения (рождения) 
синглетной пары в подсистеме коллективизированных фермионов с одновременным рождением (уничтожением) комбинированной синглетной пары, состоящей из фермиона Хаббарда и коллективизированного фермиона. Очевидно, что это взаимодействие имеет важное значение для описания сверхпроводящей фазы редкоземельных интерметаллидов.

Шесть слагаемых эффективного гамильтониана (19) имеют четвертый порядок. Слагаемое, пропорциональное матричному элементу $\eta_{q} B_{k}^{*}(f, m)$, среди прочих, описывает косвенное обменное взаимодействие локализованных электронов, через коллективизированные состояния. Следующее слагаемое, которое пропорционально $\eta_{k_{1}} C_{f}^{*}(k, q, p)$, описывает нелокальное парное взаимодействие в подсистеме коллективизированных электронов с затравочной амплитудой рассеяния, зависящей от состояния фермионов Хаббарда. Последние четыре члена в (19) возникли в результате второго унитарного преобразования. Первое из них, пропорциональное $\Delta_{f}(k, p) t_{f m}$, описывает процессы уничтожения (рождения) синглетных пар в локализованной подсистеме и рождения (уничтожения) синглетных пар в коллективизированной подсистеме. Это динамическое перемешивание синглетных пар также имеет существенное значение при описании сверхпроводящей фазы. Следующее слагаемое, пропорциональное $\Delta_{f}(k, p) \Delta_{f}^{*}\left(k_{1}, p_{1}\right)$, описывает коррелированную динамику синглетных пар коллективизированной подсистемы. Корреляция заключается в том, что амплитуда соответствующих переходов убывает по мере заполнения локализованных состояний фермионами Хаббарда. В частности, при полном заполнении локализованных состояний такие процессы полностью подавлены. Наконец, два последних слагаемых описывают обменную связь в локализованной подсистеме, а также трехцентровые взаимодействия (или коррелированные перескоки). Происхождение этих слагаемых, как уже отмечалось, аналогично их происхождению при выводе $t-J$-модели из модели Хаббарда. Обменное взаимодействие, пропорциональное $J_{f m}$, как и в теории высокотемпературной сверхпроводимости, имеет важное значение для описания конкуренции между магнитной и сверхпроводящей фазами. Трехцентровые взаимодействия, как установлено в последнее время, существенно

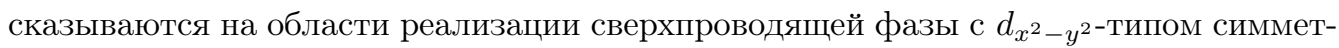
рии параметра порядка. Следует подчеркнуть, что если последнее слагаемое играет важную роль при высоком уровне заполнения локализованных состояний, то предыдущее слагаемое существенно как раз в случае слабого заполнения в локализованной подсистеме. Это означает, что необходимо учитывать оба слагаемых в совокупности для построения фазовой диаграммы, отражающей, в частности, зависимость от концентрации критической температуры перехода в сверхпроводящую фазу.

Заметим, что в полученном эффективном гамильтониане динамика синглетных пар (превращающихся при конкретных значениях импульса в куперовские пары) как в локализованной, так и в коллективизированной подсистемах управляется параметрами, имеющими высокий порядок по $V_{k}$. Это означает, что с хорошей степенью точности исследование сверхпроводящей фазы, а также конкуренции между магнитной и сверхпроводящей фазами можно проводить в рамках простейшего приближения среднего поля. Однако этот круг вопросов выходит за рамки данной ра- 
боты, и соответствующие результаты исследований будут изложены в другой статье. Здесь же мы ограничимся рассмотрением важного вопроса о величинах обменных взаимодействий в рамках полученных представлений. Знание величин обменных параметров и их зависимости от расстояния необходимо для понимания природы магнитоупорядоченной фазы.

Магнитное взаимодействие в локализованной подсистеме определяется двумя слагаемыми. Кроме предпоследнего слагаемого с параметром $J_{f m}$, обменная связь формируется еще одним слагаемым четвертого порядка. Его интенсивность зависит от $B_{k}^{*}$. После усреднения по состояниям коллективизированной подсистемы (как это обычно делается в РККИ-теории) мы получаем выражение, описывающее второе косвенное обменное взаимодействие в локализованной подсистеме, которое зависит от состояния коллективизированных электронов:

$$
H_{\mathrm{exch}}^{(2)}=\sum_{f m} I_{f m}\left(\vec{S}_{f} \vec{S}_{m}+\frac{1}{4} \widehat{N}_{f} \widehat{N}_{m}\right)
$$

где обменный интеграл

$$
I_{f m}=\frac{1}{4 N} \sum_{k} \eta_{k} B_{k}^{*}(f, m) e^{-i k m} n_{k}
$$

а $n_{k}$ - функция распределения коллективизированных электронов. Суммируя два вклада, находим, что обменное взаимодействие в локализованной подсистеме спиновых моментов описывается гамильтонианом

$$
H_{\text {exch }}=\sum_{f m}\left\{I_{f m}\left(\vec{S}_{f} \vec{S}_{m}+\frac{1}{4} \widehat{N}_{f} \widehat{N}_{m}\right)+J_{f m}\left(\vec{S}_{f} \vec{S}_{m}-\frac{1}{4} \widehat{N}_{f} \widehat{N}_{m}\right)\right\} .
$$

Величины $I_{f m}$ и $J_{f m}$ не являются независимыми, а выражаются через параметры исходной модели Андерсона, и в связи с этим для практических приложений представляется интересным провести их сравнительный анализ. С этой целью остановимся на результатах численных расчетов обменных интегралов, акцентируя внимание на зависимости обменных интегралов от расстояния между узлами $f$ и $m$ решетки. Для удобства это расстояние мы будем характеризовать номером координационной сферы $N_{\text {cs }}$. Для определенности будем считать, что ионы редкоземельных элементов расположены в узлах кубической решетки, тогда как ионы $s(p)$-элементов, формирующих зону проводимости, находятся в центрах элементарных ячеек. Как следует из выражений для $I_{f m}$ и $J_{f m}$, характер дисперсии $c$-электронов $\varepsilon_{k}$ слабо влияет на величину обменных интегралов. Поэтому при выборе $\varepsilon_{k}$ ограничимся приближением сильной связи:

$$
\varepsilon_{k}=2 t_{1}^{c}\left(\cos k_{x}+\cos k_{y}+\cos k_{z}\right)
$$

где $t_{1}^{c}$ - интеграл перескока $c$-электронов между ближайшими ионами $s(p)$-элементов. В то же время зависимость $V_{k}$ от квазиимпульса может оказаться существенной для вычисления значений обменных интегралов $I_{f m}$ и $J_{f m}$. Имея это в виду, введем два 


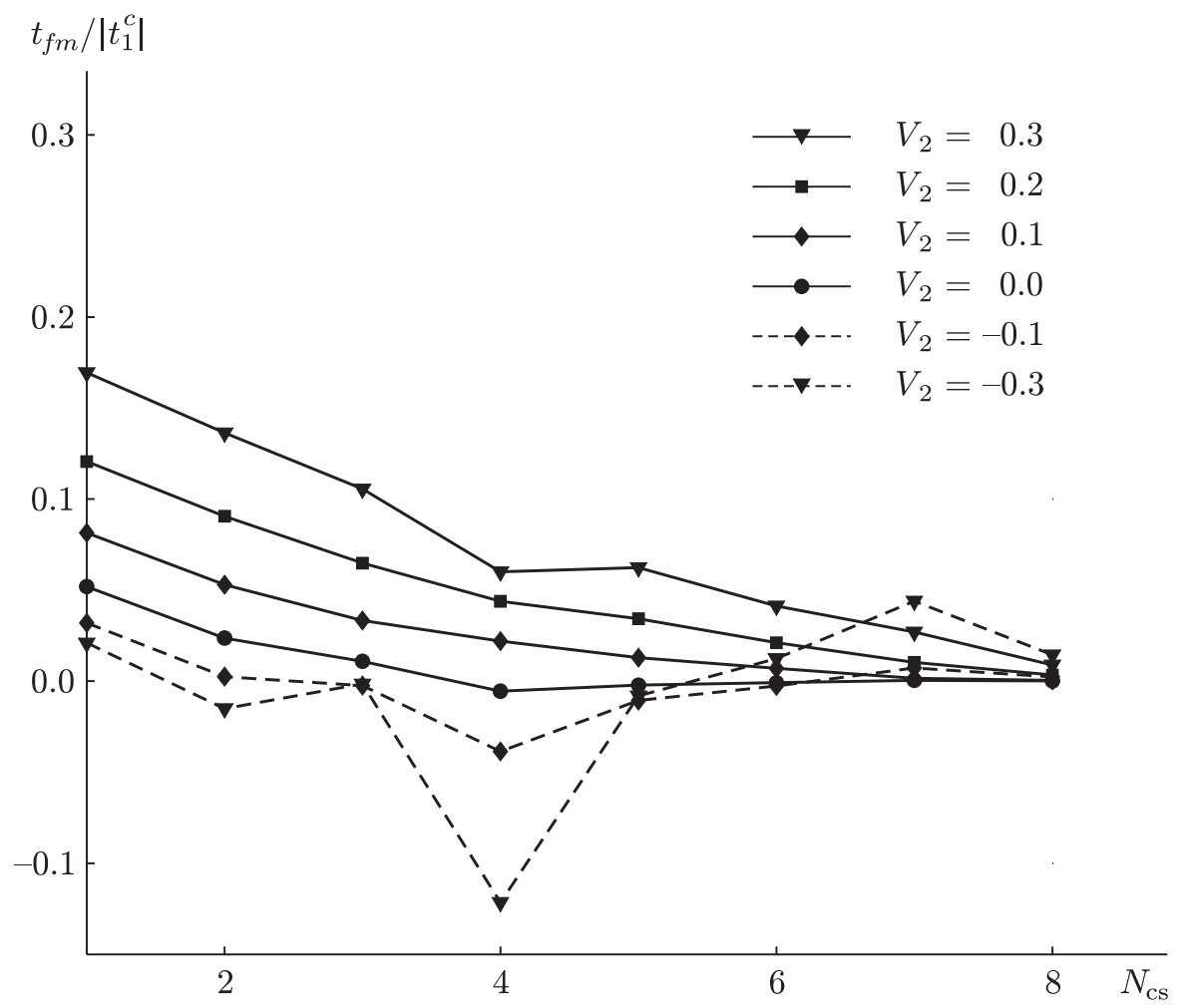

Рис. 2. Зависимость эффективного интеграла перескока от номера координационной сферы при различных значениях константы гибридизации $V_{2}$ (параметры ПАМ приведены в тексте).

параметра $V_{1}$ и $V_{2}$, позволяющие достаточно полно отразить перемешивание орбиталей $f$ - и $s(p)$-ионов. При этом для $V_{k}$ получаем следующее выражение:

$$
\begin{aligned}
& V_{k}=8 V_{1} \cos \frac{k_{x}}{2} \cos \frac{k_{y}}{2} \cos \frac{k_{z}}{2}+8 V_{2}\left(\cos \frac{3 k_{x}}{2} \cos \frac{k_{y}}{2} \cos \frac{k_{z}}{2}+\right. \\
&\left.+\cos \frac{k_{x}}{2} \cos \frac{3 k_{y}}{2} \cos \frac{k_{z}}{2}+\cos \frac{k_{x}}{2} \cos \frac{k_{y}}{2} \cos \frac{3 k_{z}}{2}\right) .
\end{aligned}
$$

На рис. 2, 3 представлены результаты численных расчетов, отражающих зависимости эффективного интеграла перескока $f$-электрона $t_{f m}$ и параметра сверхобменного взаимодействия $J_{f m}$ от номера координационной сферы $N_{\mathrm{cs}}$ при различных значениях константы гибридизации $V_{2}$. Энергетические параметры измеряются в единицах $\left|t_{1}^{c}\right|$. Вычисления проводились при следующих параметрах ПАМ: $V_{1}=0.4\left|t_{1}^{c}\right|, E_{0}=-2\left|t_{1}^{c}\right|, U=10\left|t_{1}^{c}\right|$. Из этих рисунков видно, что при положительных значениях $V_{2}$ как $t_{f m}$, так и $J_{f m}$ монотонно убывают. Абсолютная величина сверхобменного взаимодействия для ближайших соседей хорошо согласуется с экспериментально наблюдаемым значением температуры Нееля в тяжелофермионных 


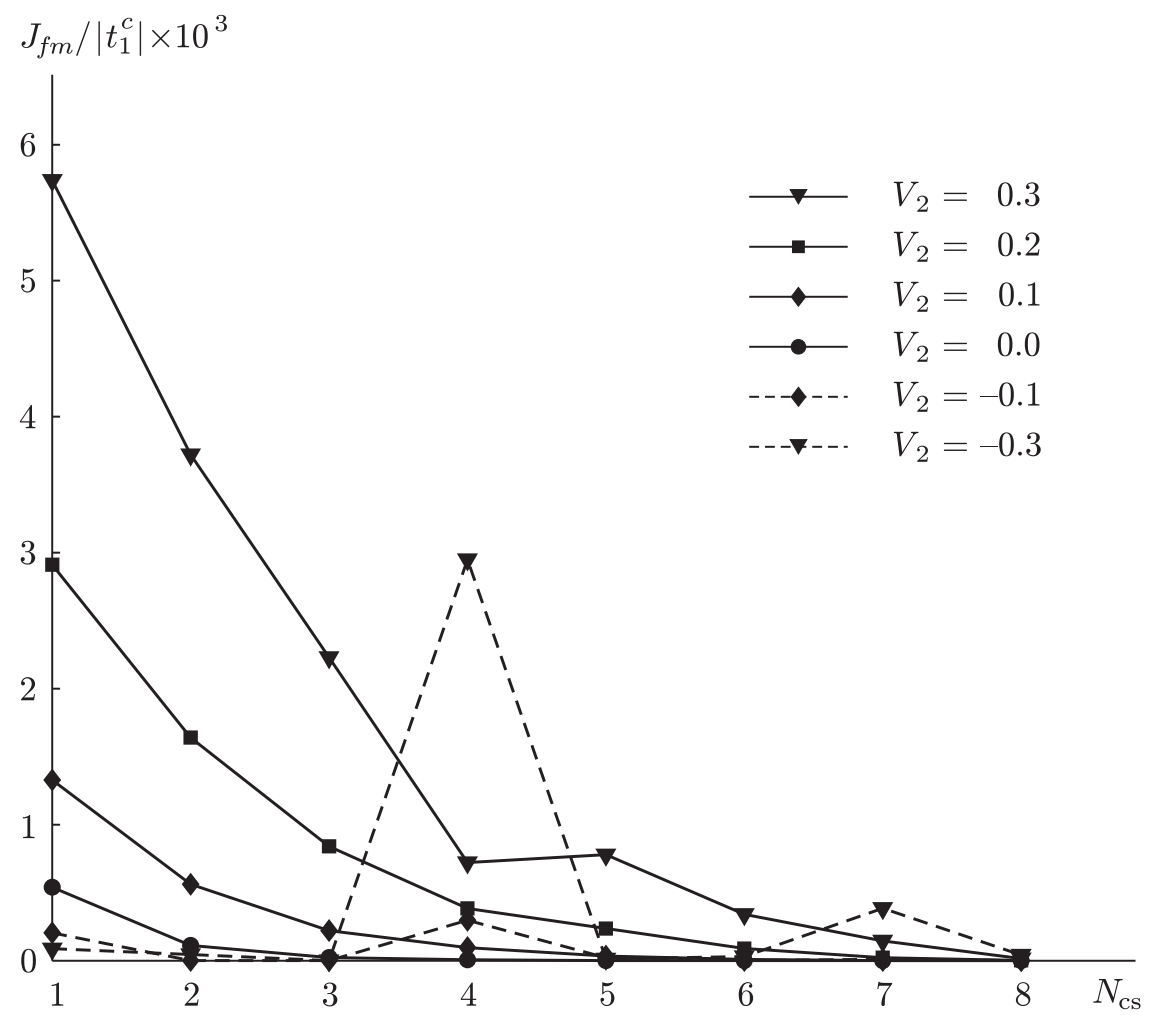

Рис. 3. Зависимость интеграла сверхобменного взаимодействия от номера координационной сферы (параметры ПАМ приведены в тексте).

системах: $T_{\mathrm{N}} \sim 10 \mathrm{~K}$. Важное свойство, вытекающее из представленных расчетов, состоит в том, что значения $J_{f m}$ для ближайших и следующих за ближайшими соседями отличаются на незначительную величину порядка 30\%. Поскольку оба значения положительны, а значит, существенны эффекты фрустрированных взаимодействий, следует ожидать подавления тенденции к магнитоупорядоченной фазе и формирования основного состояния системы локализованных спиновых моментов по типу спиновой жидкости. Интересная зависимость возникает при значении $V_{2}=-0.3\left|t_{1}^{c}\right|$, когда гибридизационная константа $V_{2}$ по абсолютной величине близка к значению $V_{1}\left(=0.4\left|t_{1}^{c}\right|\right)$, но противоположна по знаку. Как показано на рис. 3, в этом случае интеграл сверхобменного взаимодействия для четвертой координационной сферы значительно превосходит все остальные значения $J_{f m}$. Это означает, что подсистема редкоземельных ионов может быть разделена на восемь подрешеток кубической симметрии, не взаимодействующих между собой через механизм сверхобмена. Параметр подрешетки равен удвоенному параметру исходной решетки. Внутри каждой из подрешеток связи не фрустрированы, и $J_{f m}>0$. Это способствует формированию дальнего антиферромагнитного порядка. Однако окон- 


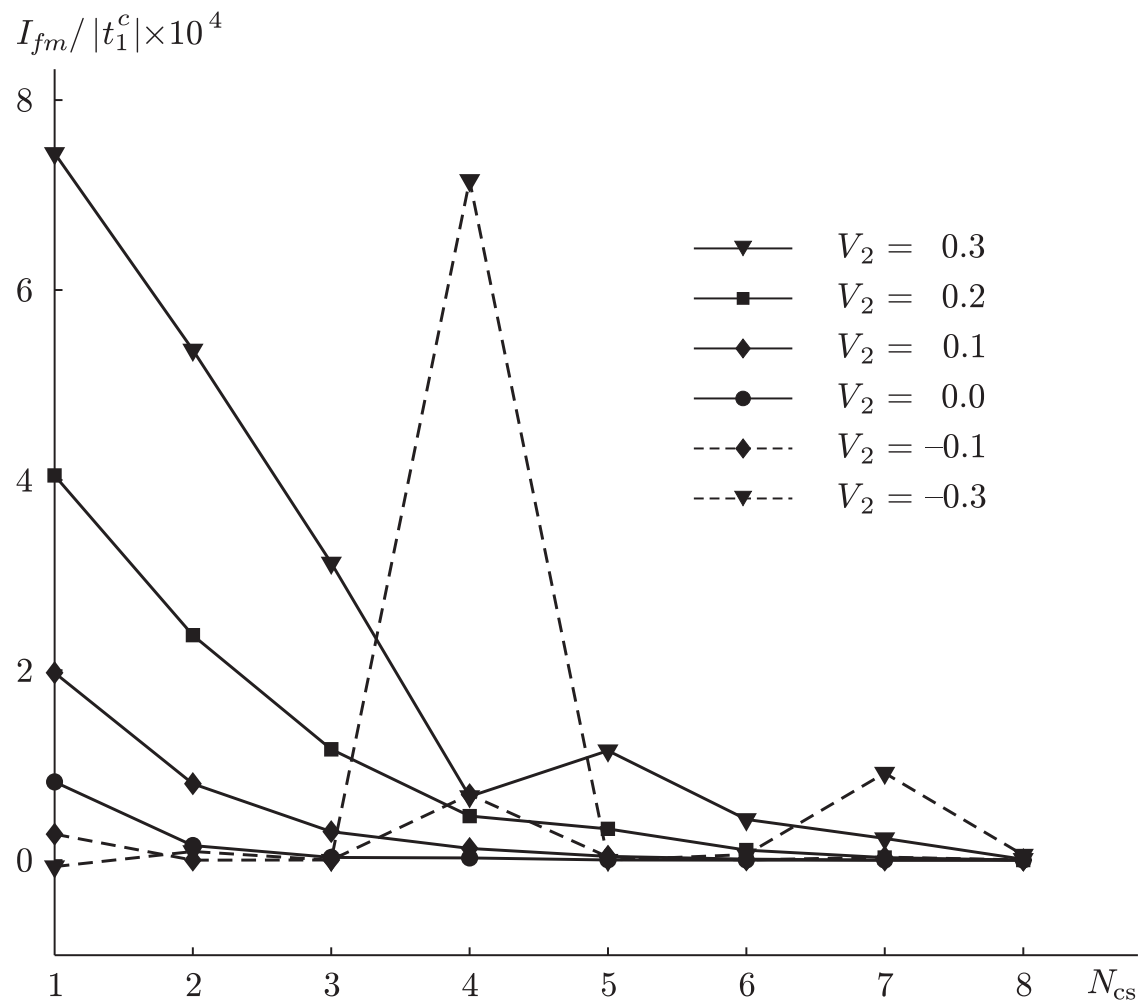

Рис. 4. Зависимость интеграла косвенного обменного взаимодействия от номера координационной сферы.

чательная спиновая конфигурация всей системы будет зависеть от других типов взаимодействий.

На рис. 4 представлены результаты расчетов величины обменного интеграла косвенного взаимодействия $I_{f m}(21)$ для различных расстояний между редкоземельными ионами. Функция распределения коллективизированных электронов $n_{k}$ для простоты аппроксимировалась фермиевской функцией с затравочным энергетическим спектром для коллективизированных электронов. Вычисления проводились при значении химического потенциала $\mu=-2\left|t_{1}^{c}\right|$. Остальные параметры ПАМ такие же, как и при расчете $I_{f m}$. Из рис. 4 видно, что интеграл косвенного обмена $I_{f m}$ уменьшается с увеличением номера координационной сферы. При этом абсолютная величина $I_{f m}$ почти на порядок меньше соответствующих значений $J_{f m}$. Поскольку интенсивность рассматриваемого косвенного обменного взаимодействия зависит от концентрации $c$-электронов, при увеличении химического потенциала значения $I_{f m}$ могут возрасти. Это можно видеть на рис. 5: значения $I_{f m}$ увеличиваются почти на порядок при заполнении зоны проводимости. В результате значения констант сверхобменного взаимодействия и косвенного взаимодействия могут быть сопоставимы по порядку величины при больших концентрациях зонных электронов. 


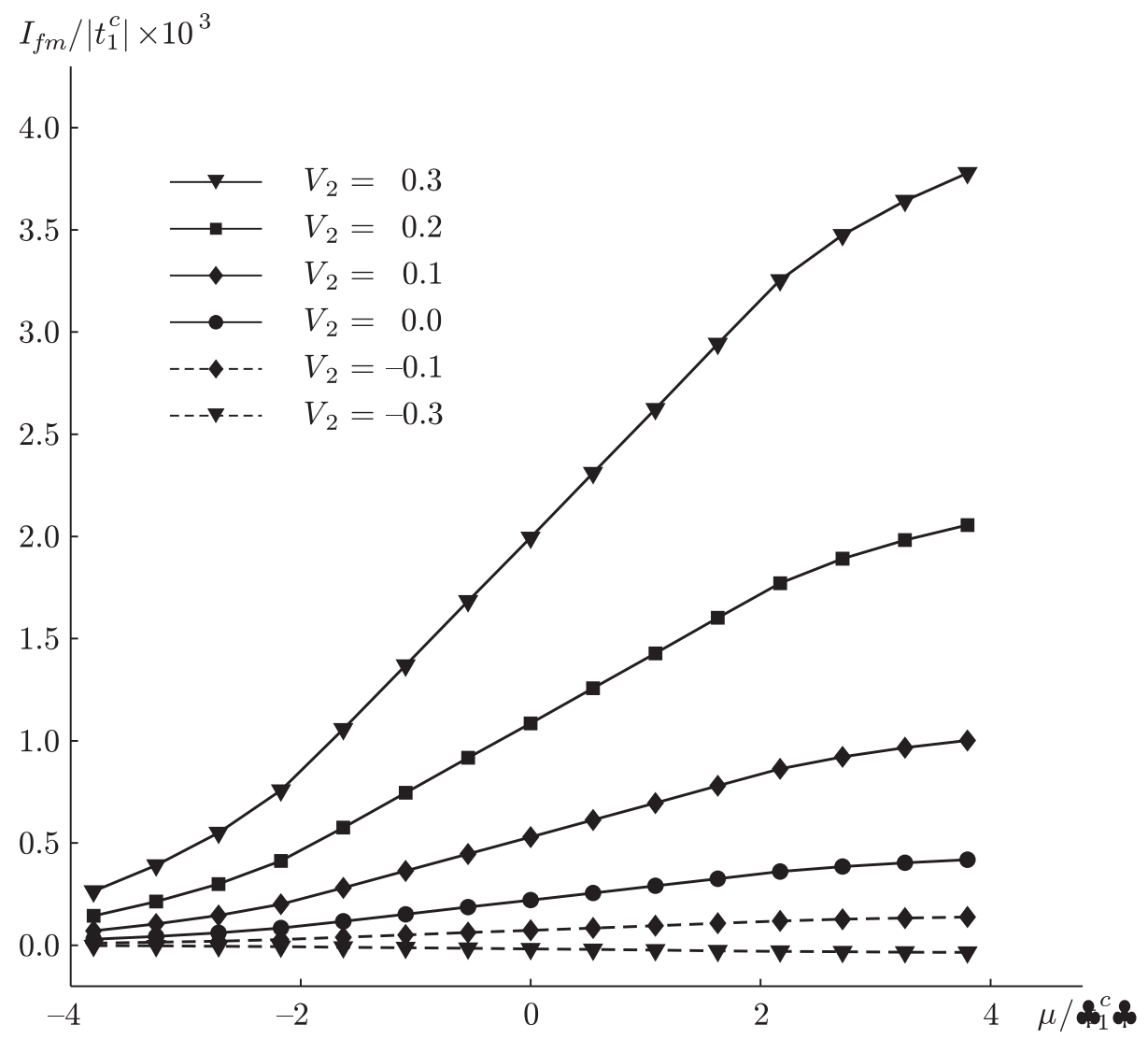

Рис. 5. Зависимость интеграла косвенного обменного взаимодействия между ближайшими соседями от химического потенциала при различных значениях константы гибридизации $V_{2}$.

Таким образом, из приведенного численного анализа двух механизмов обменного взаимодействия (косвенного обмена и сверхобмена) на основе полученного эффективного гамильтониана ПАМ следует вывод об отсутствии конкуренции между двумя отмеченными механизмами обменных взаимодействий при всех параметрах модели. Относительный вклад этих двух механизмов в формирование основного состояния системы зависит как от заселенности локализованных орбиталей, так и от степени заполнения зоны проводимости.

В заключение еще раз отметим, что ключевой особенностью предложенного способа получения $H_{\text {eff }}$ является использование параметра малости, определяемого только теми гибридизационными процессами, которые идут с участием высокоэнергетических состояний локализованной подсистемы. Соответственно, только эти процессы исключаются в первом и втором порядках с помощью унитарных преобразований. При этом процессы гибридизации в нижней хаббардовской подзоне остаются. Общей отличительной чертой упомянутых во введении способов получения $H_{\text {eff }}$ для 
ПАМ является стремление избавиться от всех гибридизационных процессов (как высокоэнергетических, так и низкоэнергетических). Причина неприменимости такого подхода в режиме смешанной валентности кроется, как уже отмечалось, в расходимости матричных элементов эффективных взаимодействий, полученных в результате интегрирования низкоэнергетических гибридизационных процессов.

Благодарности. Работа выполнена при финансовой поддержке РФФИ (грант № 07-02-00226), Программы ОФН РАН, Интеграционного проекта СО РАН.

\section{Список литературы}

[1] E. Bauer, G. Hilscher, H. Michor et al. (eds.), Proc. Int. Conf. on Strongly Correlated Electron Systems (Vienna, Austria, July 26-30, 2005), Physica B, 359-361 (2005); H. Harima, H. Kawamura, Y. Kitaoka et al. (eds.), Proc. 17th Int. Conf. on Magnetism (Kyoto, Japan, August 20-25, 2006), J. Magn. Magn. Mater., 310:2 (2007), 169-944; 945-1882; $1883-2892$.

[2] Ю. А. Изюмов, Н. И. Чащин, Д. С. Алексеев, Теория сильно коррелированных систем. Метод производящего функционала, РХД, М.-Ижевск, 2006.

[3] C. M. Varma, Comments Solid State Phys., 11 (1985), 221.

[4] K. A. Kikoin, M. N. Kiselev, A. S. Mishchenko et al., Phys. Rev. B, 59:23 (1999), 15070-15084.

[5] Y. Bang, Mixed moment wave function for magnetic heavy fermion compounds, arXiv: cond-mat/0206247.

[6] C. Pépin, Phys. Rev. Lett., 98 (2007), 206401; Kondo breakdown as a selective Mott transition in the Anderson lattice, arXiv: cond-mat/0610846.

[7] J. R. Schrieffer, P. A. Wolff, Phys. Rev., 149:2 (1966), 491-492.

[8] C. Lacroix, M. Cyrot, Phys. Rev. B, 20:5 (1979), 1969-1976.

[9] C. Proetto, A. López, Phys. Rev. B, 24:6 (1981), 3031-3036.

[10] Д. Ф. Дигор, П. Ентел, М. Маринаро и др., ТМФ, 127:2 (2001), 304-316.

[11] A. Hübsch, K. W. Becker, Eur. Phys. J. B, 52:3 (2006), 345-353.

[12] L.-J. Zhou, Q.-Q. Zheng, J. Magn. Magn. Mater., 109:2-3 (1992), 237-242.

[13] С. В. Тябликов, Методы квантовой теории магнетизма, Наука, М., 1965.

[14] P. Fulde, Electron Correlations in Molecules and Solids, Springer Ser. Solid-State Sci., 100, Springer, Berlin, 1995.

[15] Ю. А. Изюмов, УФН, 167:5 (1997), 465-497. 